\title{
Vulnerabilidad del Cutzamala
} Fecha de publicación: 16 de mayo de 2021
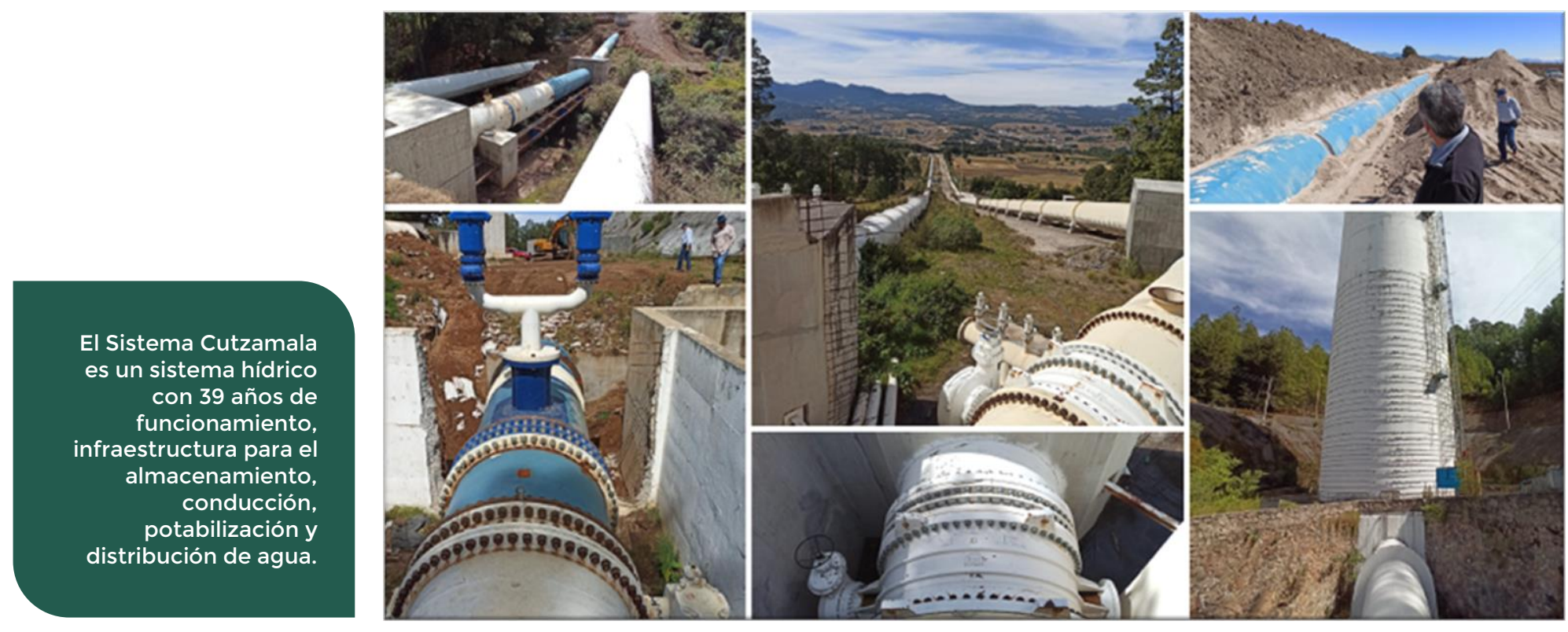

El Sistema Cutzamala es una de las fuentes de abastecimiento de agua potable más grandes del país, -

ya que aprovecha las aguas de la cuenca alta del río Cutzamala, que provienen de las presas Tuxpan y El Bosque, en el estado de Michoacán, y Colorines, Ixtapan del Oro, Villa Victoria, Valle de Bravo, y Chilesdo en el Estado de México.

Debido a la sobreexplotación de las cuencas de los valles de México y Toluca-Ixtlahuaca, se hizo necesario captar el agua de cuencas externas diferentes a la del Alto Lerma, que desde la década de 1950 ya presentaba signos de sobreexplotación, por lo que, en 1982, el Sistema Cutzamala inicia su operación.

En su primera etapa de operación se aportaron $4 \mathrm{~m}^{3} / \mathrm{s}$ de la presa Villa Victoria, y se construyó la planta de bombeo Núm. 5, así como la subestación eléctrica para la misma.

En su segunda etapa de operación, en 1985, se incrementó el suministro a $10 \mathrm{~m}^{3} / \mathrm{s}$, con agua de la presa Valle de Bravo a la planta potabilizadora Los Berros, para lo cual se construyeron las plantas de bombeo 2,3 y 4 . 
Autor: Rodrigo Ulises Santos Téllez, Raúl Medina Mendoza y José

La tercera etapa está integrada por los subsistemas Chilesdo y Colorines, para un aprovechamiento total de $9 \mathrm{~m} 3 / \mathrm{s}$. El primero se encuentra en operación desde enero de 1993. Este capta las aguas del río Malacatepec en la presa Chilesdo, con lo cual se evita que escurran hasta la presa Colorines, reduciéndose así los costos de operación.

De las 7 presas que integran el Sistema Cutzamala, 3 son de almacenamiento:

- Tuxpan

- Ixtapan del Oro

- Colorines

y 4 son derivadoras:

- $\quad$ El Bosque

- Valle de Bravo

- Villa Victoria

- Chilesdo

Además, cuenta con la siguiente infraestructura:

- 6 macroplantas de bombeo

- $\quad 205.70$ kilómetros de tuberías de acero y concreto con diámetros entre 1.07 y 3.5 metros.

- $\quad 72.55$ kilómetros de canales abiertos

- 44 kilómetros de túneles.

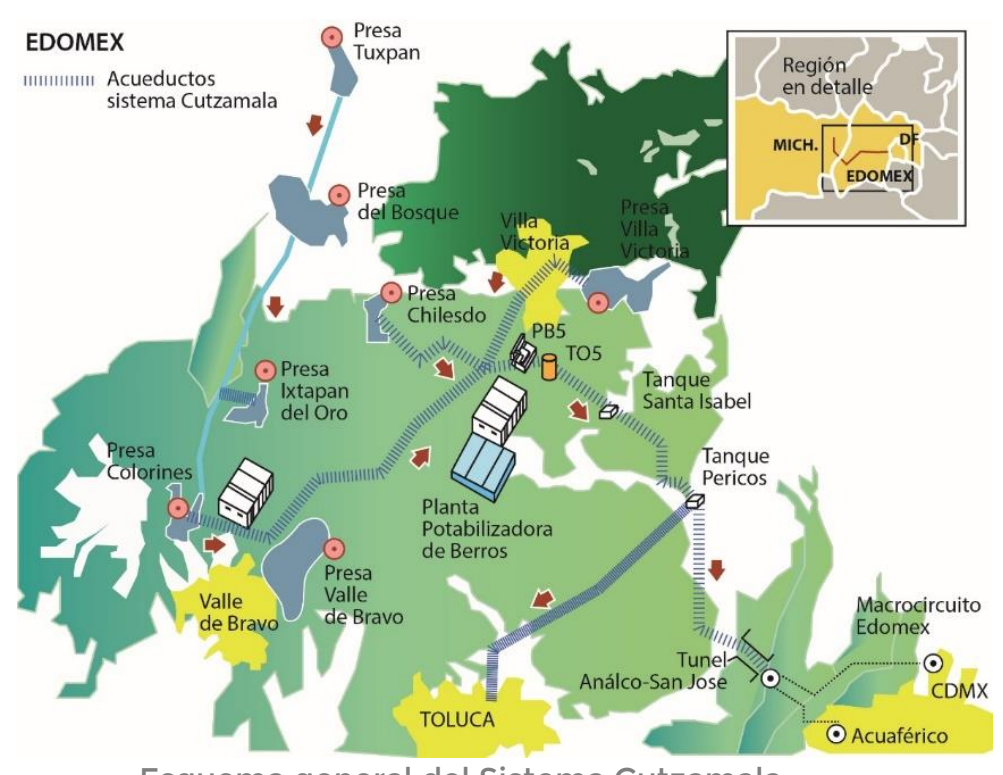

Esquema general del Sistema Cutzamala

El Sistema Cutzamala es un sistema hídrico con 39 años de funcionamiento, infraestructura para el almacenamiento, conducción, potabilización y distribución de agua potable para la población e industria de la Ciudad de México y el Estado de México, de este último las ubicadas en las zonas centrales de la cuenca de México y el valle de Toluca. Es considerada una de las mayores obras de 
ingeniería civil en el mundo, ya que debe bombearse el agua desde una altitud de 1,600 m s. n. m., en su punto más bajo, hasta los $2,702 \mathrm{~m}$ s. $\mathrm{n}$. m., en su punto más alto. Este sistema se extiende por las entidades de Michoacán, el Estado de México y la Ciudad de México.

El Sistema Cutzamala aporta el $26 \%$ del agua potable que se consume en el valle de México. El resto del suministro se obtiene del Sistema Lerma, de pozos, y de diversos manantiales que operan con normalidad.

El crecimiento de la población en la zona metropolitana de la Ciudad de México y del valle de Toluca, la consecuente demanda de agua potable y la falta de fuentes alternas de suministro de agua a incorporar en el corto plazo son las causas que motivan mantener operando en condiciones adecuadas esta infraestructura, para evitar conflictos sociales y garantizar un abastecimiento continuo en cantidad y calidad.

Sin embargo, como toda obra de infraestructura, esta debe recibir mantenimiento preventivo periódico, y se deben atender situaciones de emergencia que pongan en riesgo el abastecimiento y su integridad. Para realizar estas acciones, el Organismo de Cuenca Aguas del Valle de México (Ocavam) debe realizar operativos de mantenimiento programados que obligan a detener el suministro durante un corto periodo, que puede ser de unas horas hasta tres días. Por supuesto esto causa inconformidad por parte de los habitantes, complica las acciones de suministro de los organismos operadores y genera presión por parte de todos los involucrados para reiniciar el suministro de agua potable. Por tales motivos, los operativos de mantenimiento se reducen a las actividades mínimas necesarias, lo cual incrementa el riesgo de una falla mayor en el corto o mediano plazo. Esta situación aumenta la vulnerabilidad del sistema, principalmente de la planta Los Berros hasta los puntos de entrega en la Ciudad de México y el Estado de México.

Como puede verse en la imagen, de presentarse una falla en algún punto del sistema, antes de la planta potabilizadora de Los Berros, este tiene la capacidad de mitigar temporalmente el problema, ya que se conforma de tres grupos de presas; por tanto, en caso de perderse el suministro de alguna de ellas se tiene la posibilidad de no parar el suministro y, en el peor de los escenarios, solo disminuirlo temporalmente.

Sin embargo, a partir de la planta de Los Berros, el sistema no tiene redundancia ni flexibilidad, ya que solo cuenta con una línea de conducción entre la planta de bombeo 5 (PB5) y la torre de oscilación 5 (TO5), y las líneas L1 y L2 conducen de forma simultánea el caudal a entregar. Por tanto, una falla en cualquier punto entre la planta potabilizadora de Los Berros y la TO5 obligaría a un paro completo del sistema, y si ocurriese una falla entre la TO5 y el túnel Analco San José, obligaría a reducir el suministro al $50 \%$. Otro punto vulnerable del sistema es el propio túnel Analco San José, ya que, de presentarse una falla en este, se reduciría el suministro a un $35 \%$.

Para mitigar este riesgo, la Conagua, desde hace unos años, construye la línea 2 de alta presión entre la PB5 y la TO5 y la línea 3 entre la TO5 y el Túnel Analco San José, para tener flexibilidad de operación. Esta nueva infraestructura no tiene el objetivo de aumentar el caudal entregado, su principal aporte es contar con una conducción alterna que permita dar mantenimiento al sistema sin necesidad de detener el suministro, con lo cual los operativos de mantenimiento preventivo y emergente se podrán realizar de forma completa y adecuada, sin afectar a la población. 
Autor: Rodrigo Ulises Santos Téllez, Raúl Medina Mendoza y José

Manuel Rodríguez Varela

DOI: doi.org/10.24850/b-imta-perspectivas-2021-18

Actualmente ya se tiene en operación un tramo de 17 km de la línea 3, entre El Salitre y el tanque Pericos, y en la tercera semana de marzo entró en operación la línea 2 de alta presión, la cual se espera que esté terminada para finales de 2021.

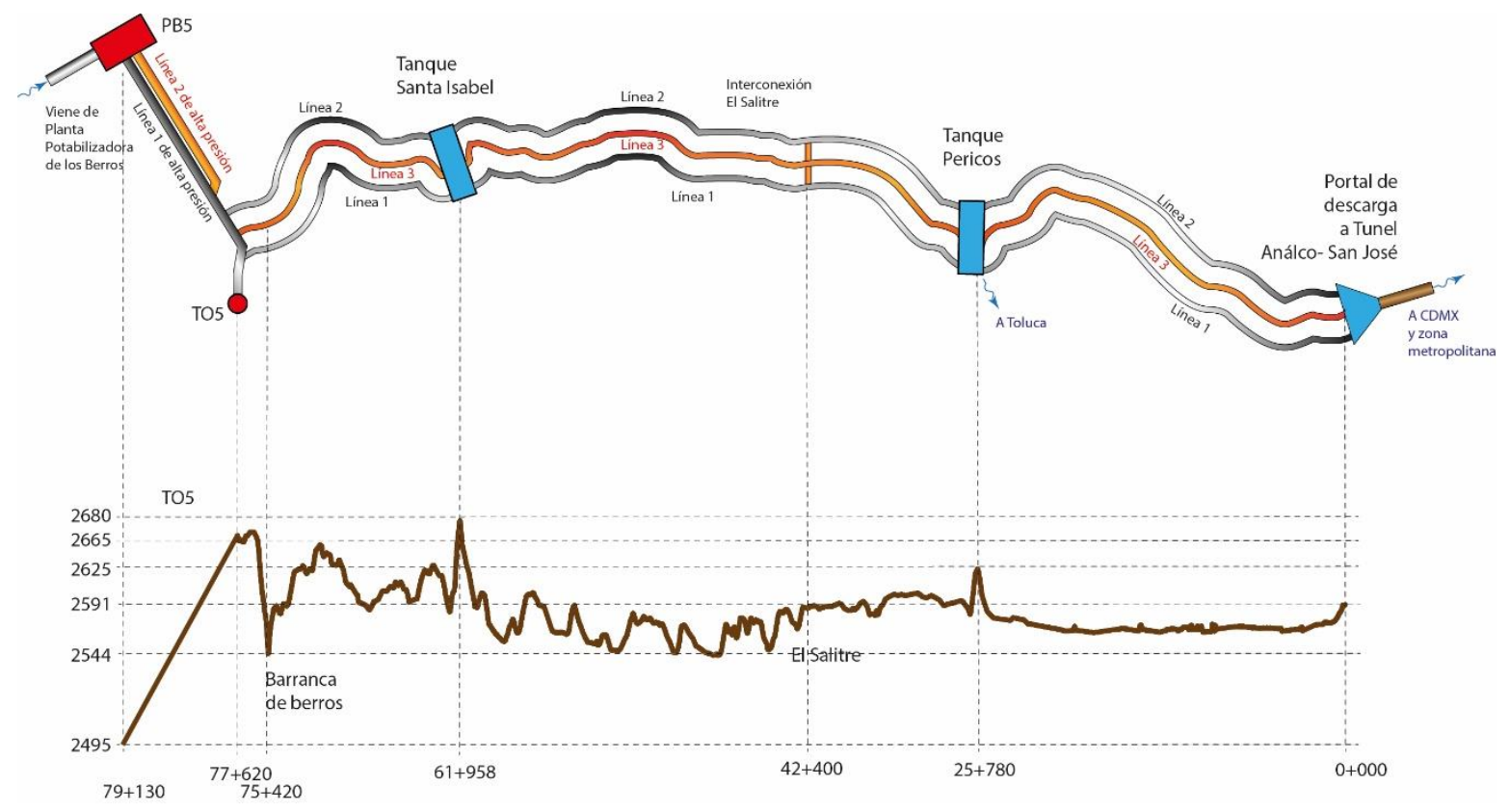

Esquema del tramo PB5-TASJ

No obstante, esta situación no resuelve toda la problemática del sistema Cutzamala, también se debe trabajar en la modernización de la planta potabilizadora de Los Berros y la conducción entre esta y la PB5.

La situación que pone en grave riesgo el suministro de agua potable desde el sistema Cutzamala es la pérdida de capacidad de captación de las cuencas de aporte a las presas. Durante el presente año, el sistema de presas se encuentra en su nivel más bajo en los últimos 25 años, lo cual está asociado a la sequía de intensidades de D2 y D3 en las cuencas de aporte, y para los meses de mayo y junio se anticipa una grave escasez de agua en las presas del sistema. 


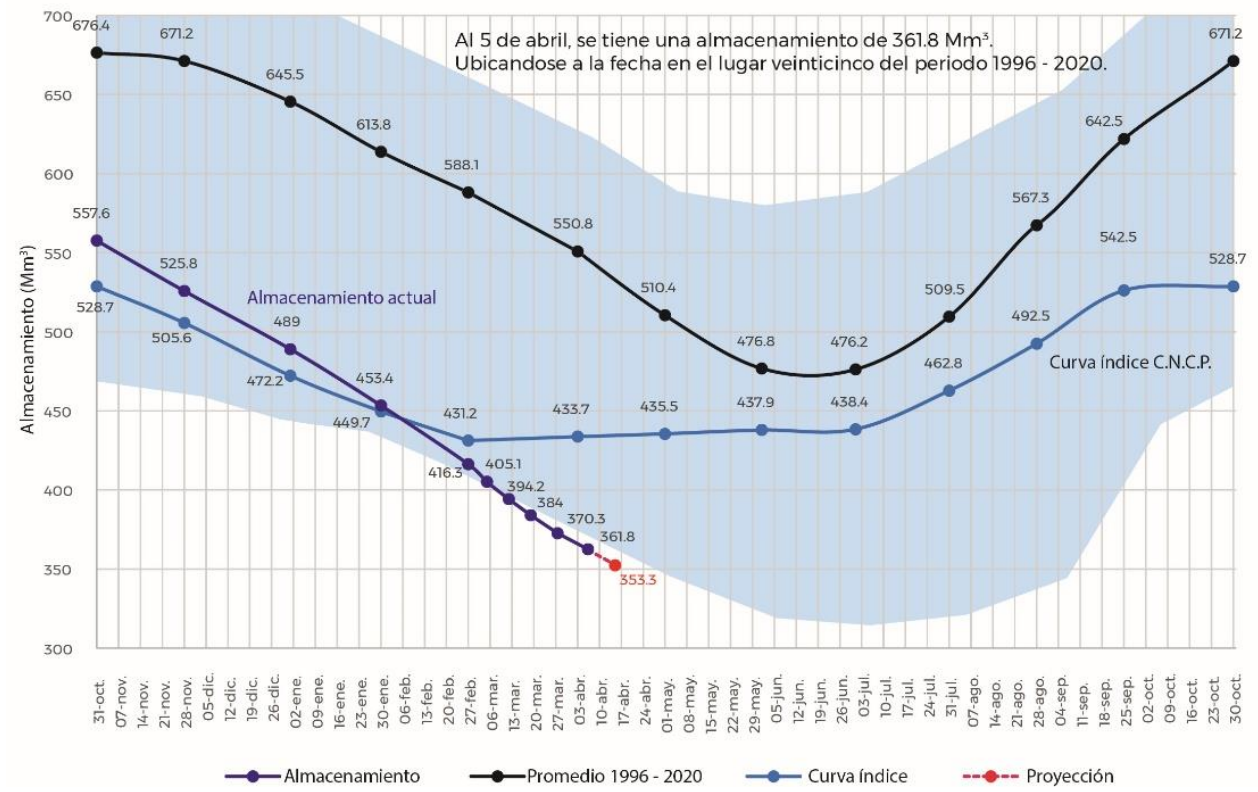

Almacenamiento del sistema de presas del sistema Cutzamala al 3 de mayo de 2021 (Comisión Nacional del Agua, 2021)

Debido a esta situación, la Conagua, en acuerdo con el Sistema de Aguas de la Ciudad de México y la Comisión del Agua del Estado de México, ha establecido una política de reducción de extracciones desde el inicio del periodo de estiaje, con lo que se busca garantizar el abasto durante 2021 y 2022. Por tanto, si bien se ha avanzado significativamente en brindar la infraestructura necesaria para garantizar el suministro desde el Sistema Cutzamala, se requiere tomar acciones para recuperar las cuencas de aporte y garantizar en el agua necesaria en las presas, y dichas acciones deben ser coordinadas por las diversas instituciones y los tres órdenes de gobierno, así como por las poblaciones que reciben el suministro y las que se encuentran dentro de las cuencas.

Las acciones de intervención en las porciones medias y altas de las cuencas del Sistema Cutzamala, para incrementar la disponibilidad del agua de forma sostenible, deben realizarse de forma participativa con los productores que usufructúan los recursos naturales para satisfacer sus necesidades básicas. El manejo de su territorio lo realizan mayormente con prácticas tradicionales, que son inadecuadas y no sostenibles. Asimismo, la ocupación productiva de los terrenos se hace de una forma contraria a la propia vocación o aptitud de los suelos -como la agricultura en laderas de fuertes pendientes-, generando un "conflicto de uso" que se traduce en la degradación paulatina de los recursos naturales con impactos en la calidad y cantidad del recurso hídrico.

Así, para que sea exitoso cualquier programa cuyo objetivo sea el de incrementar la disponibilidad del recurso hídrico en las cuencas del Sistema Cutzamala, se deberá buscar armonizar los intereses y necesidades que giran en torno a resolver la sostenibilidad a largo plazo de los recursos naturales y asociados, evitando la degradación de suelo, agua, vegetación y fauna y, a la vez, cumplir con los objetivos de los productores agropecuarios y forestales. Entonces, por un lado, están los dueños o usufructuarios de las parcelas (impactos in situ), interesados en mantener o mejorar su capital de recursos naturales para una producción sostenida, que les permita asegurar como mínimo su autoconsumo y quienes aspiran a obtener ingresos económicos que les garanticen su sustentabilidad 
y la de sus familias en el largo plazo. Y por el otro lado, los impactos que ocurren aguas abajo (impactos ex situ) en cuerpos de agua e infraestructura, motivo de preocupación y atención de las diferentes instancias de gobierno y autoridades que manejan el recurso hídrico en el Sistema Cutzamala.

Las tecnologías a implementar para resolver la problemática y alcanzar la sostenibilidad de los recursos naturales en las cuencas son abundantes y se seleccionan en función de las características específicas de cada unidad de producción y condición socioeconómica de los productores.

Actualmente se enfatizan las soluciones basadas en la naturaleza (SBN), que en el ámbito de las cuencas se conocen como mejores prácticas, tecnologías conservacionistas de agua y suelo o prácticas vegetativas. Para las cuencas del Sistema Cutzamala una acción estratégica lo constituye la SBN de "producir agua", término que engloba el proteger y recuperar las áreas vitales para la existencia del agua, como son: zonas de infiltración; áreas de recarga acuífera; nacientes, lagos, arroyos, ríos y quebradas con tecnologías específicas de desarrollo que integran la repoblación forestal focalizada hacia las zonas de recarga inmediata; el tratamiento y reúso de aguas residuales que permitan recuperar volúmenes; la cosecha de lluvia para la producción alimentaria que integre la ocupación del suelo por capacidad de uso, con lo que se contrarrestan los conflictos de uso de los terrenos; el diseño hidrológico del terreno o técnica Keyline, y la labranza mínima o cero labranza. En el mismo sentido, la captación de agua de lluvia de los techos se considera como una acción para producir agua en las cuencas.

La implementación en campo de las mejores prácticas requiere pasar por un proceso de planificación en tres ámbitos territoriales de intervención:

1) A nivel de unidad de producción, planificando de forma participativa y acompañada por los dueños de las fincas, quienes deben ser los verdaderos tomadores de las decisiones a partir de las propuestas de mejores prácticas, utilizando para este proceso una herramienta metodológica de Plan Participativo de Producción y Conservación (PPPC).

El principio de trabajo en este ámbito territorial es que una finca con mejores prácticas no contribuirá al resto de la cuenca con contaminantes en forma de escurrimientos excesivos, azolves, basura y agroquímicos.

2) A nivel de microcuenca hidrográfica o unidad de escurrimiento para contrarrestar el fenómeno erosivo que ocurre dentro del territorio conformado por una o varias comunidades, en cárcavas, caminos, cortes de carretera, riberas de arroyos, zonas de riego, las cuales por su magnitud y extensión sobrepasan los límites de las fincas y, por ende, la capacidad de solución de un productor o grupo de productores, necesitándose la intervención y apoyo de una o varias instituciones. Para este ámbito territorial se recomienda utilizar la herramienta Plan de Conservación y Manejo Participativo de Microcuencas (PCMPM). El principio de trabajo en este ámbito es que la sumatoria de las microcuencas con buenas prácticas de manejo posibilitarán que cada subcuenca alcance un desarrollo equilibrado.

3) A nivel de las cuencas de aportación, integrando los planes por microcuenca y definiendo las políticas generales que sirvan como marco de conducción de las intervenciones gubernamentales, coordinando los esfuerzos y dándole un sentido de dirección a los programas federalizados y a los llevados a cabo por los gobiernos estatales y municipales. 
Autor: Rodrigo Ulises Santos Téllez, Raúl Medina Mendoza y José

Manuel Rodríguez Varela

DOI: doi.org/10.24850/b-imta-perspectivas-2021-18

En este nivel territorial es donde se posibilita la evaluación de los impactos de las mejores prácticas en la cantidad y calidad del agua que llega a las presas que abastecen al Sistema Cutzamala.

Las acciones en las porciones medias y altas de las cuencas del Sistema Cutzamala requieren ineludiblemente de la intervención simultánea y coordinada de las diferentes instancias y entidades de todos los órdenes de gobierno, tanto de las que tienen injerencia en los recursos naturales como de las que administran directamente el recurso hídrico.

\section{Bibliografía}

Comisión Nacional del Agua (4 de mayo de 2021). Sesión Ordinaria 1491 del Comité Nacional de Grandes Presas. Ciudad de México. 